\title{
Partial Realization in Dynamic Justification Logic
}

\author{
Samuel Bucheli, Roman Kuznets ${ }^{\star \star}$, and Thomas Studer \\ Institut für Informatik und angewandte Mathematik, Universität Bern \\ Bern, Switzerland \\ \{bucheli, kuznets, tstuder\}@iam.unibe.ch
}

\begin{abstract}
Justification logic is an epistemic framework that provides a way to express explicit justifications for the agent's belief. In this paper, we present OPAL, a dynamic justification logic that includes term operators to reflect public announcements on the level of justifications. We create dynamic epistemic semantics for OPAL. We also elaborate on the relationship of dynamic justification logics to Gerbrandy-Groeneveld's PAL by providing a partial realization theorem.
\end{abstract}

\section{Introduction}

Public announcement logic $[14,13]$ describes how public announcements affect an agent's belief (knowledge). It is a subarea of dynamic epistemic logic [18], which studies the relationship between belief (knowledge) and communication in general. The effect of a public announcement of statement $A$ is represented by a formula $[A] B$ that means $B$ holds after the public announcement of $A$. It is generally assumed that announcements do not affect the material reality of the world, but that the agent trusts the announced facts, partly because their verification may not be an option unless the agent is omniscient. In this paper, we consider beliefs rather than knowledge, hence we concentrate on GerbrandyGroeneveld's logic PAL.

The idea of justification logic [2] is to formalize reasons for the agent's belief. Instead of $\square A$ used in the modal language to state that the agent believes $A$, in justification logic, a specific reason for belief is given $-t: A$ says that the agent believes $A$ for reason $t$. This ability to express explicit reasons for the agent's belief provides a novel approach to several problems of multi-agent systems and formal epistemology. Justifications can be employed, for instance, to tackle the logical omniscience problem [4], to analyze several classical epistemic puzzles [2, $3]$, and to study common knowledge $[1,8]$.

It is natural to ask how public announcements factor into the reasoning of the agent. Public announcement logic describes how the beliefs change, but not why. The aim of this paper is to suggest ways of formalizing the answer to this why. The postulate of Gerbrandy-Groeneveld's PAL that deals with belief change is

$$
\square(A \rightarrow[A] B) \leftrightarrow[A] \square B .
$$

\footnotetext{
* Supported by Swiss National Science Foundation grant 200021-117699.

** Supported by Swiss National Science Foundation grant PZ00P2_131706.
} 
To understand its meaning, it is better to read the equivalence as two separate implications. From left to right, the postulate says that an agent who believes that $B$ must be the case whenever a true fact $A$ is announced will in fact believe $B$ after an actual announcement of $A$. For instance, elite-level frequent flyers can usually check in for their flight at the business counter by presenting their elite membership card, which can also be attached to their luggage to make public their elite status. This rule should be known to airline employees. The left-toright implication then means that, when Ann presents her elite membership card to Bob at the business counter, he would know he should check her in. If we are to convert this implication from a statement about beliefs to a statement about reasoning, the result should be something like

$$
t:(A \rightarrow[A] B) \rightarrow[A] s: B,
$$

where $t$ represents the airline's regulations regarding business-counter check-in procedures and $s$ is the reason why Bob starts checking Ann in. There are three possibilities how $s$ can be related to $t$ : (A) $s=t$, where the regulations themselves tell Bob to check Ann in; (B) $s=\Uparrow t$, where $\Uparrow$ represents the inference Bob has to make from the regulations after the elite card is shown; and (C) $s=\Uparrow_{A} t$, where the inference process explicitly mentions both regulations $t$ and the demonstration of Ann's elite card $A$. In principle, any of the three options can be used to model the situation.

In our joint work with Bryan Renne and Joshua Sack [7], we developed the logic JPAL based on option (A). In this paper, we will present a new logic OPAL based on option (B). We should, therefore, start by explaining why the simplicity of JPAL may not always be sufficient. Imagine that Ann has been upgraded to business class (say, as a reward for postponing her original flight, which was overbooked). So, according to the same regulations, she can check in with Bob based on her ticket alone without announcing her elite status: i.e., $t: B$. But Ann may choose to announce her elite status anyways: i.e., $[A] t: B$. In JPAL, where $s=t$, after the elite status is announced, $t$ encodes two different reasons for Bob to check Ann in. By contrast, in OPAL, these two reasons will be represented by two different terms, $t$ and $\Uparrow t$, of which the latter depends on Ann's elite status while the former is due to the ticket alone. In this situation, Bob would want to distinguish between the two reasons because of the difference in baggage allowances: an elite frequent flyer is often allowed to check more luggage for free than an owner of a business class ticket that has been upgraded from economy.

In addition, in this and similar cases, the JPAL approach implies that the meaning of the regulations changes after public announcements: if Ann has an economy ticket, the regulations do not allow her a business-counter check-in until she shows her elite card, and then they do. This is a little counterintuitive since the regulations are a legal document whose meaning should not be changed by each public announcement. The use of reason $\Uparrow t$ enables us to separate the permanent status of the regulations from their momentary applications influenced by public announcements.

It may seem that adding the exact content of the public announcement to the term $\Uparrow_{A} t$, as in option $(\mathrm{C})$, provides even more information than the fact of 
a public announcement in $\Uparrow t$. However, mixing formulas and terms makes the syntax unnecessarily complicated. Worse than that, it makes schematic reasoning, i.e., reasoning invariant with respect to substitutions, impossible. Indeed, the update axiom (1) is actually an axiom scheme that does not depend on the announcement. Therefore, the operation that represents the update on the term level perhaps should not depend on the announcement either. We will, therefore, not consider option $(\mathrm{C})$ in this paper.

One might even consider a fourth option where the reason $s$ is the announcement $A$ itself: i.e., $s=\Uparrow_{A}$. But the only statement that the announcement of $A$ can plausibly support by itself is $A$, in which case it should always support it. This would be incompatible with PAL since not all formulas there are successful.

Let us now look at the other implication - from right to left - and see how options (A) and (B) manifest themselves there. The implication states that an agent who will believe $B$ after an announcement of $A$ must believe that, if $A$ is true and announced, $B$ holds after the announcement. For instance, if Charlie, while standing in a long line at the economy check-in counter, sees Ann showing her elite card and being served by Bob at the business counter, $[A] \square B$, then Charlie has empirical evidence $e$ that Ann is served at the business counter, $[A] e: B$. It would be natural for Charlie to believe that having an elite status and showing it gets one to the business counter, $\square(A \rightarrow[A] B)$. But it seems even clearer in this case that Charlie's empirical observation $e$ cannot explain the causality of the implication $A \rightarrow[A] B$. If before Ann showed up, Charlie had read the sign that invited elite members to the business counter, then Charlie's memory of this sign, refreshed by Ann's actions, could serve as such an explanation. Thus, instead of using $e$, as in JPAL, in this example it also seems better to use $\Downarrow e$, where $\Downarrow$ is yet another new operation of our logic OPAL.

Apart from the already mentioned [7], not much work has been done so far to combine justification logic and dynamic epistemic logic. A notable exception is Renne's research about introducing new evidence [15] and eliminating unreliable evidence [16] in the framework of justification logic. He also studied the effect certain announcements have on the expressivity of justification logic [17]. However, the modal logic counterparts of those systems do not correspond to any traditional public announcement logic. Both JPAL from [7] and OPAL introduced here are intended as justification logics with public announcement operators whose belief dynamics closely corresponds to the modal belief dynamics of Gerbrandy-Groeneveld's PAL [13].

In this paper, we introduce a justification logic OPAL, operational public announcement logic, that extends the language of JPAL with unary operations $\Uparrow$ and $\Downarrow$ to express the occurrence of public announcements and the corresponding change in the justifications of the agent's belief. We present OPAL axiomatically in Sect. 2 and provide it with dynamic epistemic semantics in Sect. 3. After recalling the logic PAL in Sect. 4, in Sect. 5 we prove the soundness and completeness of the newly introduced OPAL with respect to the presented semantics and compare this proof with that in PAL. 
While both JPAL and OPAL are intended as exact justification counterparts of PAL, this has to be formally established via the so-called realization theorem. An idea for proving the realization theorem for PAL was sketched in [7], naturally for the logic JPAL discussed there. This idea was dependent on certain properties of JPAL that were conjectured to hold. In Sect. 6 of this paper, we implement this idea and discuss its application to both JPAL and OPAL. The implementation has turned out to be far from being straightforward. In particular, for JPAL, we have established only a partial realization theorem, one that provides a method of substituting terms for $\square$ 's in any theorem of PAL that contains no $\square$ 's within announcements. In contrast to most known constructive proofs of realization theorems, our method, sketched in [7] and implemented here, is not based on a cut-free sequent calculus for PAL. Instead, we first use a modal reduction of PAL to a simpler logic K4, then use the standard realization method for the latter, and finally reverse the performed reduction on the justification side. This reversal process employs the replacement techniques developed by Fitting [11]. The closest analog of this method can be found in [10], where Fitting realizes S5 by reducing it to $\mathrm{K} 45$. However, there the reversal of the reduction is trivial. Extending this method to OPAL presented unexpected challenges, which we also discuss at the end of Sect. 6.

Most of the proofs are relegated to the appendix for space considerations.

\section{Justification Logic}

The language of OPAL extends the language typically used in justification logic by adding public announcement formulas $[A] B$ and two unary operations on terms, $\Uparrow$ and $\Downarrow$, to express the update dynamics of evidence.

Definition 1 (OPAL Language). We fix countable sets Cons of constants, Vars of variables, and Prop of atomic propositions. The language of OPAL consists of the terms $t \in \mathrm{Tm}$ and the formulas $A \in \mathrm{Fml}_{\mathrm{J}}$ formed by the grammar

$$
\begin{aligned}
t::=x|c|(t \cdot t)|(t+t)| ! t|\Uparrow t| \Downarrow t & & x \in \text { Vars, } c \in \text { Cons } \\
A::=p|\neg A|(A \rightarrow A)|t: A|[A] A & & p \in \text { Prop }
\end{aligned}
$$

Notation 2 ( $\sigma$-Sequences) $\sigma$ and $\tau$ (with and without subscripts) will denote finite sequences of formulas. $\varepsilon$ denotes the empty sequence. Given such a sequence $\sigma=\left(A_{1}, \ldots, A_{n}\right)$ and a formula $B$, the formula $[\sigma] B$ is defined as follows:

$$
[\sigma] B:=\left[A_{1}\right] \ldots\left[A_{n}\right] B \text { if } n>0 \quad \text { and } \quad[\sigma] B:=B \text { if } n=0 .
$$

Further, sequences $\sigma, B:=\left(A_{1}, \ldots, A_{n}, B\right)$ and $B, \sigma:=\left(B, A_{1}, \ldots, A_{n}\right)$. For another sequence $\tau=\left(C_{1}, \ldots, C_{m}\right)$, we define $\tau, \sigma:=\left(C_{1}, \ldots, C_{m}, A_{1}, \ldots, A_{n}\right)$.

Definition 3 (OPAL Deductive System). The axioms of OPAL consist of all $\mathrm{Fml}_{\mathrm{J}}$-instances of the following schemes:

1. $[\sigma] A$, where $A$ is a classical propositional tautology 


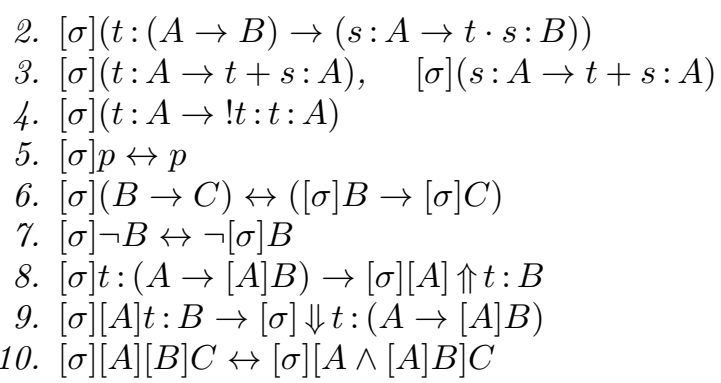

(application)

(sum)

(introspection)

(independence)

(normality)

(functionality)

(update $\Uparrow$ )

(update $\Downarrow)$

(iteration)

The deductive system OPAL is a Hilbert system that consists of the above axioms of OPAL and the following rules of modus ponens (MP) and axiom necessitation $(A N)$ :

$$
\frac{A \quad A \rightarrow B}{B}(M P), \quad \frac{c_{1}, \ldots, c_{n} \in \text { Cons } C \text { is an OPAL-axiom }}{\left[\sigma_{1}\right] c_{1}: \cdots:\left[\sigma_{n}\right] c_{n}: C} \text { (AN), }
$$

where $\sigma_{i}$ 's are (possibly empty) finite sequences of formulas.

The following example gives some intuition as to how updates are represented by the operations on evidence.

Example 4. For any $p \in$ Prop and any $c_{1}, c_{2} \in$ Cons, we have $\vdash[p] \Uparrow\left(c_{1} \cdot c_{2}\right): p$.

Proof. We use PR to denote the use of propositional reasoning.

1. $c_{1}:(([p] p \leftrightarrow p) \rightarrow(p \rightarrow[p] p))$ AN for the tautology $([p] p \leftrightarrow p) \rightarrow(p \rightarrow[p] p)$

2. $c_{2}:([p] p \leftrightarrow p)$

3. $\left(c_{1} \cdot c_{2}\right):(p \rightarrow[p] p)$

4. $[p] \Uparrow\left(c_{1} \cdot c_{2}\right): p$

AN for the independence axiom $[p] p \leftrightarrow p$

from 1 and 2 by application and PR

from 3 by update $\Uparrow$ and $\mathrm{PR}$

Note that $p$ need not, in general, be true. The presence of $\Uparrow$ in the term $\Uparrow\left(c_{1} \cdot c_{2}\right)$ clearly signifies that this evidence for $p$ is contingent on a prior public announcement. However, the exact content of such a public announcement, $p$ in our case, is not recorded in the term. This design decision enables us to avoid the overcomplexification of the language and is similar to the introspection operation in the traditional justification logics: ! $t$ is evidence for $t: A$ whenever $t$ is evidence for $A$; however, the formula $A$ is not recorded in the term ! $t$ either.

Remark 5. The announcement-free fragment of OPAL (the first four axioms with $\sigma=\varepsilon$, rule MP, and rule $\mathrm{AN}$, restricted to $c_{n}: C$ ) is the well-known justification logic J4 (see [5]).

We will consider not only OPAL but also JPAL (see [7] for details), which does not include the two term operations $\Uparrow$ and $\Downarrow$.

Definition 6 (JPAL Deductive System). The axioms of JPAL are the axioms of OPAL where the two update axiom schemes are replaced by the single scheme

$$
[\sigma] t:(A \rightarrow[A] B) \leftrightarrow[\sigma][A] t: B .
$$


The deductive system JPAL is a Hilbert system that consists of the axioms of JPAL and the rules (MP) and $(A N)$, where the formula $C$ in $(A N)$ now stands for an axiom of JPAL.

The following lemma states a standard property of justification logics that holds for OPAL and JPAL; it can be proved by an easy induction on the length of derivation.

Lemma 7 (Lifting). If $s_{1}: B_{1}, \ldots, s_{m}: B_{m}, C_{1}, \ldots, C_{n} \vdash A$, then there is a term $t\left(s_{1}, \ldots, s_{m}, y_{1}, \ldots, y_{n}\right)$ such that

$$
s_{1}: B_{1}, \ldots, s_{m}: B_{m}, y_{1}: C_{1}, \ldots, y_{n}: C_{n} \vdash t\left(s_{1}, \ldots, s_{m}, y_{1}, \ldots, y_{n}\right): A
$$

for fresh variables $y_{1}, \ldots, y_{n}$.

Corollary 8 (Constructive Necessitation). For any formula $A$, if $\vdash A$, then there is a ground term $t$ such that $\vdash t: A$.

\section{Semantics}

We adapt the Kripke-style semantics for Justification Logic due to Fitting [9] . A similar semantics for JPAL was presented in [7]. Our semantics uses Kripke models augmented by an evidence function that relates each world-term pair $(w, t)$ to a set of formulas $\mathcal{E}(w, t)$ that the term $t$ can justify at the world $w$.

Definition 9 (K4-Frame). A K4-frame is a pair $(W, R)$ that consists of a set $W \neq \emptyset$ of (possible) worlds and of a transitive accessibility relation $R \subseteq W \times W$.

Definition 10 (Evidence Function). An evidence function on a K4-frame $(W, R)$ is a function $\mathcal{E}: W \times \mathrm{Tm} \rightarrow \mathcal{P}\left(\mathrm{Fml}_{\mathrm{J}}\right)$ that satisfies the following closure conditions:

1. Monotonicity: if $R(w, v)$, then $\mathcal{E}(w, t) \subseteq \mathcal{E}(v, t)$ for any $t \in \mathrm{Tm}$.

2. Axioms: if $c: A$ is derivable by the $A N$-rule, then $A \in \mathcal{E}(w, c)$ for any $w \in W$.

3. Application: if $(A \rightarrow B) \in \mathcal{E}(w, t)$ and $A \in \mathcal{E}(w, s)$, then $B \in \mathcal{E}(w, t \cdot s)$.

4. Sum: $\mathcal{E}(w, s) \cup \mathcal{E}(w, t) \subseteq \mathcal{E}(w, s+t)$ for any $s, t \in \operatorname{Tm}$ and any $w \in W$.

5. Introspection: if $A \in \mathcal{E}(w, t)$, then $t: A \in \mathcal{E}(w, ! t)$.

In a model of OPAL, there is an evidence function $\mathcal{E}^{\sigma}$ for each finite sequence $\sigma$ of formulas. The idea is that the evidence function $\mathcal{E}^{\sigma}$ models the "evidential situation" that arises after the formulas in $\sigma$ have been publicly announced.

Definition 11 (OPAL Model). $A$ model is a structure $\mathcal{M}=(W, R, \mathcal{E}, \nu)$, where $(W, R)$ is a $\mathrm{K} 4$-frame, $\nu:$ Prop $\rightarrow \mathcal{P}(W)$ is a valuation, and function $\mathcal{E}$ maps finite sequences $\sigma$ of formulas to evidence functions $\mathcal{E}^{\sigma}$ on $(W, R)$ and satisfies

$$
\begin{gathered}
A \rightarrow[A] B \in \mathcal{E}^{\sigma}(w, t) \text { implies } B \in \mathcal{E}^{\sigma, A}(w, \Uparrow t), \\
B \in \mathcal{E}^{\sigma, A}(w, t) \text { implies } A \rightarrow[A] B \in \mathcal{E}^{\sigma}(w, \Downarrow t), \\
\mathcal{E}^{\sigma, A, B}(w, t)=\mathcal{E}^{\sigma, A \wedge[A] B}(w, t) .
\end{gathered}
$$


Conditions (3), (4), and (5) correspond to the update axiom $\Uparrow$, the update axiom $\Downarrow$, and the iteration axiom respectively.

Definition 12 (Truth in OPAL Models). A ternary relation $\mathcal{M}, w \Vdash A$ for formula $A$ being satisfied at a world $w \in W$ in a model $\mathcal{M}=(W, R, \mathcal{E}, \nu)$ is defined by induction on the structure of $A$ :

$-\mathcal{M}, w \Vdash p$ if and only if $w \in \nu(p)$.

- Boolean connectives behave classically.

$-\mathcal{M}, w \Vdash t: A$ if and only if 1) $A \in \mathcal{E}^{\varepsilon}(w, t)$ and 2) $\mathcal{M}, v \Vdash A$ for all $v \in W$ with $R(w, v)$.

$-\mathcal{M}, w \Vdash[A] B$ if and only if $\mathcal{M}_{A}, w \Vdash B$, where $\mathcal{M}_{A}=\left(W_{A}, R_{A}, \mathcal{E}_{A}, \nu_{A}\right)$ is defined as follows: $W_{A}:=W ; R_{A}:=\{(s, t) \mid R(s, t)$ and $\mathcal{M}, t \Vdash A\}$; $\left(\mathcal{E}_{A}\right)^{\sigma}:=\mathcal{E}^{A, \sigma} ;$ and $\nu_{A}:=\nu$. Note that $\mathcal{M}_{A}$ is indeed a model: $R_{A}$ is transitive, $\left(\mathcal{E}_{A}\right)^{\sigma}$ is an evidence function on $\left(W_{A}, R_{A}\right)$ for each $\sigma$, and $\mathcal{E}_{A}$ satisfies conditions (3)-(5) from Def. 11.

We write $\mathcal{M} \Vdash A$ to mean that $\mathcal{M}, w \Vdash A$ for all $w \in W$. We say that formula $A$ is valid, written $\Vdash A$, to mean that $\mathcal{M} \Vdash A$ for all models $\mathcal{M}$. For a sequence $\tau=\left(A_{1}, \ldots, A_{n}\right)$ of formulas we use $\mathcal{M}_{\tau}=\left(W_{\tau}, R_{\tau}, \mathcal{E}_{\tau}, \nu_{\tau}\right)$ to denote the model $\left(\cdots\left(\left(\mathcal{M}_{A_{1}}\right)_{A_{2}}\right) \cdots\right)_{A_{n}}$. Note that $\left(\mathcal{E}_{\tau}\right)^{\sigma}=\mathcal{E}^{\tau, \sigma} ;$ in particular, $\left(\mathcal{E}_{\tau}\right)^{\varepsilon}=\mathcal{E}^{\tau}$.

Our notion of model is non-empty as the following example shows.

Example 13. We define the structure $\mathcal{M}=(W, R, \mathcal{E}, \nu)$ as follows: $W:=\{w\}$; $R:=\{(w, w)\} ; \mathcal{E}^{\sigma}(w, t):=\mathrm{Fml}_{\mathrm{J}}$ for all $\sigma$ and all $t \in \mathrm{Tm}$; and $\nu(p):=\{w\}$ for all $p \in$ Prop. It is easy to see that $\mathcal{M}$ is a model.

To illustrate how the semantics works, we prove a semantic version of the result from Example 4.

Example 14. For any $p \in$ Prop and any $c_{1}, c_{2} \in$ Cons, we have $\Vdash[p] \Uparrow\left(c_{1} \cdot c_{2}\right): p$.

Proof. Let $\mathcal{M}=(W, R, \mathcal{E}, \nu)$ be an arbitrary model and let $w \in W$. By Def. 10.2, we have $([p] p \leftrightarrow p) \rightarrow(p \rightarrow[p] p) \in \mathcal{E}^{\varepsilon}\left(w, c_{1}\right)$ and $([p] p \leftrightarrow p) \in \mathcal{E}^{\varepsilon}\left(w, c_{2}\right)$. Thus, $(p \rightarrow[p] p) \in \mathcal{E}^{\varepsilon}\left(w, c_{1} \cdot c_{2}\right)$ by Def. 10.3. So, by condition (3) from Def. 11, we have $p \in \mathcal{E}^{p}\left(w, \Uparrow\left(c_{1} \cdot c_{2}\right)\right)$. Since $R_{p}(w, v)$ implies $\mathcal{M}, v \Vdash p$, i.e., $v \in \nu(p)=\nu_{p}(p)$, we have $\mathcal{M}_{p}, w \Vdash \Uparrow\left(c_{1} \cdot c_{2}\right): p$ by Def. 12 and, hence, $\mathcal{M}, w \Vdash[p] \Uparrow\left(c_{1} \cdot c_{2}\right): p$.

\section{Modal Public Announcement Logic}

In this section, we recall some of the basic definitions and facts concerning the Gerbrandy-Groeneveld modal logic of public announcements [12, 13, 18].

Definition 15 (PAL Language). The language of PAL consists of the formulas $A \in \mathrm{Fml}_{\square,[\cdot]}$ formed by the grammar

$$
A::=p|\neg A|(A \rightarrow A)|\square A|[A] A \quad p \in \text { Prop }
$$

The language $\mathrm{Fml}_{\square}$ of modal formulas without announcements is obtained from the same grammar without the $[A]$ A constructor. 
The Gerbrandy-Groeneveld theory PAL of Public Announcement Logic uses the language $\mathrm{Fml}_{\square,[\cdot]}$ to reason about belief change and public announcements.

Definition 16 (PAL Deductive System). The axioms of PAL consist of all

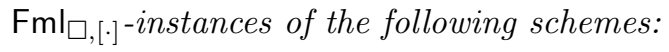

1. Axiom schemes for the modal logic $\mathrm{K} 4$

2. $[A] p \leftrightarrow p$

3. $[A](B \rightarrow C) \leftrightarrow([A] B \rightarrow[A] C)$

4. $[A] \neg B \leftrightarrow \neg[A] B$

5. $[A] \square B \leftrightarrow \square(A \rightarrow[A] B)$

6. $[A][B] C \leftrightarrow[A \wedge[A] B] C$

(independence) (normality)

(functionality)

(update)

(iteration)

The deductive system PAL is a Hilbert system that consists of the above axioms of PAL and the following rules of modus ponens (MP) and necessitation $(N)$ :

$$
\frac{A \quad A \rightarrow B}{B}(M P), \quad \frac{A}{\square A}(N) .
$$

We write $\mathrm{PAL} \vdash A$ to state that $A \in \mathrm{Fml}_{\square,[\cdot]}$ is a theorem of PAL.

We sometimes use some of the same names for both axioms of OPAL and axioms of PAL because it will always be clear from the context which of the two is meant. As before, the axioms of independence, normality, functionality, update, and iteration are called the announcement axioms.

PAL, like many traditional modal public announcement logics, features the so-

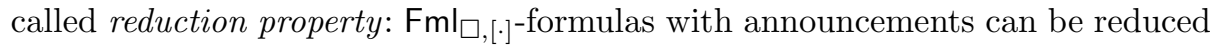
to provably equivalent $\mathrm{Fml}_{\square}$-formulas without announcements $[12,13,18]$. That means one can express what the situation is after an announcement by saying what the situation was before the announcement. The following lemma formally describes this reduction procedure (for a proof, see, for instance, [18]). This method was first introduced by Plaza in [14].

Definition 17 (Reduction). The reduction function red : Fml $\square,[\cdot] \rightarrow \mathrm{Fml}_{\square}$ is defined as follows:

1. $\operatorname{red}(p)=p$

2. red commutes with the connectives $\neg, \rightarrow$, and $\square$.

3. $\operatorname{red}([A] p)=p$.

4. $\operatorname{red}([A] \neg B)=\operatorname{red}(\neg[A] B)$.

5. $\operatorname{red}([A](B \rightarrow C))=\operatorname{red}([A] B \rightarrow[A] C)$.

6. $\operatorname{red}([A] \square B)=\operatorname{red}(\square(A \rightarrow[A] B))$.

7. $\operatorname{red}([A][B] C)=\operatorname{red}([A \wedge[A] B] C)$.

Lemma 18 (Provable Equivalence of Reductions). For all $A \in \mathrm{Fml}_{\square,[\cdot]}$, we have PAL $\vdash A \leftrightarrow \operatorname{red}(A)$.

Remark 19. The above lemma facilitates a completeness proof for PAL by reducing it to completeness of K4. Suppose that $A \in \mathrm{Fml}_{\square,[\cdot]}$ is valid. Then $\operatorname{red}(A)$ is also valid by the soundness of PAL and Lemma 18. Since $\operatorname{red}(A)$ is a formula of $\mathrm{Fml} \square$, we get by the completeness of $\mathrm{K} 4$ that $\mathrm{K} 4 \vdash \operatorname{red}(A)$ and, hence, that PAL $\vdash \operatorname{red}(A)$ because PAL extends K4. Applying Lemma 18 again, we conclude that PAL $\vdash A$. 


\section{Soundness and Completeness for OPAL}

In this section, we establish soundness and completeness of OPAL. First, soundness is shown in the usual way by induction on the length of the derivation.

Lemma 20 (Soundness). For all formulas $A$, we have that $\vdash A$ implies $\Vdash A$.

The traditional modal logic reduction approach (see Remark 19) to establishing completeness is not possible in the presence of justifications since the replacement property does not hold in Justification Logic (see [11, Sect. 6] for a detailed discussion of the replacement property in Justification Logic). That means, in particular, that $\vdash A \leftrightarrow B$ does not imply $\vdash t: A \leftrightarrow t: B$, which would be an essential step in the proof of an OPAL-analog of Lemma 18. Thus, it is not possible to transfer the completeness of J4 (see [9]) to OPAL. We will, instead, provide a canonical model construction to prove the completeness of OPAL.

Definition 21 (Maximal Consistent Sets). A set $\Phi$ of $\mathrm{Fml}_{\mathrm{J}}$-formulas is called consistent if $\Phi \nvdash A$ for some formula $A$. A set $\Phi$ is called maximal consistent if it is consistent but has no consistent proper extensions.

It can be easily shown that maximal consistent sets contain all axioms of OPAL and are closed under modus ponens and axiom necessitation.

Definition 22 (Canonical Model). The canonical model $\mathcal{M}=(W, R, \mathcal{E}, \nu)$ is defined as follows:

1. $W:=\left\{w \subseteq \mathrm{Fml}_{\mathrm{J}} \mid w\right.$ is a maximal consistent set $\}$,

2. $R(w, v)$ if and only if for all finite sequences $\sigma$ and all $t \in \mathrm{Tm}$, we have $[\sigma] t: A \in w$ implies $[\sigma] A \in v$,

3. $\mathcal{E}^{\sigma}(w, t):=\left\{A \in \mathrm{Fml}_{\jmath}:[\sigma] t: A \in w\right\}$,

4. $\nu(p):=\{w \in W: p \in w\}$.

Lemma 23 (Truth Lemma). Let $\mathcal{M}$ be the canonical model. For all formulas $D$ and all worlds $w$ in $\mathcal{M}$, we have $D \in w$ if and only if $\mathcal{M}, w \Vdash D$.

As usual, the Truth Lemma implies completeness, which, as a corollary, yields announcement necessitation.

Theorem 24 (Completeness). OPAL is sound and complete: that is, for all formulas $A \in \mathrm{Fml}$, we have $\vdash A$ if and only if $\Vdash A$.

Corollary 25 (Announcement Necessitation). Announcement necessitation is admissible: that is, for all formulas $A, B \in \mathrm{Fml}_{\text {J }}$, we have $\vdash A$ implies $\vdash[B] A$. 


\section{Forgetful Projection and Realization}

This section deals with the relationship between PAL and dynamic justification logics. It is easy to show that the forgetful projection $A^{\circ}$ of an OPAL theorem $A$ is a theorem of PAL. This means that for any theorem $A$ of OPAL, if we replace each term in $A$ with $\square$, then the resulting formula $A^{\circ}$ is a theorem of PAL. A similar result holds for JPAL [7].

Definition 26 (Forgetful Projection). The mapping $\circ: \mathrm{Fml}_{\jmath} \rightarrow \mathrm{Fml}_{\square,[\cdot]}$ is defined as follows:

$$
\begin{aligned}
p^{\circ} & =p \text { for all } p \in \text { Prop }, & & \circ \text { commutes with connectives } \neg \text { and } \rightarrow, \\
(t: A)^{\circ} & =\square A^{\circ} & & ([A] B)^{\circ}=\left[A^{\circ}\right] B^{\circ} .
\end{aligned}
$$

Theorem 27 (Forgetful Projection of OPAL). For all formulas $A \in \mathrm{Fml}_{\text {, }}$ we have OPAL $\vdash A \Longrightarrow \mathrm{PAL} \vdash A^{\circ}$.

A much more difficult question is whether a dynamic justification logic, such as JPAL or OPAL, can realize PAL: that is, whether for any theorem $A$ of PAL, it is possible to replace each $\square$ in $A$ with some term such that the resulting formula is a dynamic justification validity.

In the remainder of this paper we present the first realization technique for dynamic justification logics and establish a partial realization result for JPAL: JPAL can realize formulas $A$ that do not contain $\square$ operators within announcements. Our main idea is to reduce realization of PAL to realization of K4. In our proof, we rely on notions and techniques introduced by Fitting [11].

Definition 28 (Substitution). A substitution is a mapping from variables to terms. If $A$ is a formula and $\sigma$ is a substitution, we write A $\sigma$ to denote the result of simultaneously replacing each variable $x$ in $A$ with the term $x \sigma$.

Lemma 29 (Substitution Lemma). For every formula $A$ of $\mathrm{Fml}_{\mathrm{J}}$ and every substitution $\sigma$, we have $\vdash A$ implies $\vdash A \sigma$.

In most justification logics, in addition to this substitution of proof terms for proof variables, the substitution of formulas for propositions is also possible (see [2]). However, the latter type of substitution typically fails in logics with public announcements, as it does in both JPAL and OPAL.

Definition 30 (Annotations). An annotated formula is a modal formula in which each modal operator is annotated by a natural number. An annotated formula is properly annotated if $\square_{2 k}$ 's occur in it only in negative positions, $\square_{2 k+1}$ 's occur only in positive positions, and no $\square_{i}$ occurs twice. Positions within an announcement $[A]$ are considered neither positive nor negative: i.e., the parity of indices within announcements in properly annotated formulas is not regulated. If $A^{\prime}$ is the result of replacing all indexed modal operators $\square_{i}$ with $\square$ in a (properly) annotated formula $A$, then $A$ is called a (properly) annotated version of $A^{\prime}$. 
Definition 31 (Realization Function). $A$ realization function $r$ is a mapping from natural numbers to terms such that $r(2 i)=x_{i}$, where $x_{1}, x_{2}, \ldots$ is a fixed enumeration of all variables. For a realization function $r$ and an annotated formula $A, r(A)$ denotes the result of replacing each indexed modal operator $\square_{i}$ in $A$ with the term $r(i)$. For instance, $r\left(\square_{i} B\right)=r(i): r(B)$. A realization function $r$ is called non-self-referential on variables over $A$ if, for each subformula $\square_{2 i} B$ of $A$, the variable $r(2 i)=x_{i}$ does not occur in $r(B)$.

The following realization result for the logic K4 is due to Brezhnev [5]; the additional result about non-self-referentiality can be read off from the proof presented in [6].

Theorem 32 (Realization for K4). If $A$ is a theorem of K4, then for any properly annotated version $B$ of $A$, there is a realization function $r$ such that $r(B)$ is provable in J4. Additionally, $r$ is non-self-referential on variables over $B$.

In order to formulate the replacement theorem for JPAL, a technical result necessary for demonstrating the partial realization theorem for JPAL, we use the following convention: whenever $D(q)$ and $A$ are formulas in the same language, $D(A)$ is the result of replacing all occurrences of the proposition $q$ in $D(q)$ with $A$.

For the rest of this section, we consider only formulas $A$ that do not contain modal operators within announcements: i.e., if $[B] C$ is a subformula of $A$, then $B$ does not contain modal operators.

We will use a theorem that was first formulated by Fitting [11] for LP. A closer look at its proof shows that it also holds in our context. Moreover, while Fitting's formulation only mentions replacement at a positive position, adapting the formulation and the proof to replacement at a negative position is quite easy. The following is an instance of the extended formulation:

Theorem 33 (Replacement for JPAL). Assume the following:

1. $D(q)$ is a properly annotated formula in which the proposition $q$ occurs once and outside of announcements. $A$ and $D(q)$ share no annotations, and $B$ and $D(q)$ share no annotations. If $q$ occurs positively in $D(q)$, then $A$ and $B$ are properly annotated formulas; if $q$ occurs negatively, then $\neg A$ and $\neg B$ are.

2. $r_{1}$ is a realization function that is non-self-referential on variables over $D(A)$ and $D(B)$.

3. If $q$ occurs positively in $D(q)$, then JPAL $\vdash r_{1}(A) \rightarrow r_{1}(B)$. If $q$ occurs negatively, then JPAL $\vdash r_{1}(B) \rightarrow r_{1}(A)$.

Then there is some realization/substitution pair $\langle r, \sigma\rangle$ such that

$$
\text { JPAL } \vdash r_{1}(D(A)) \sigma \rightarrow r(D(B))
$$

and $r$ is non-self-referential on variables over $D(B)$.

We now have all ingredients ready to establish our realization theorem. The following diagram shows how we obtain it. We start with a formula $A \in \mathrm{Fml}_{\square,[\cdot]}$. Using reduction, K4 realization from Theorem 32, and the replacement theorem, we construct a formula $r(A) \in \mathrm{Fml}_{\mathrm{J}}$ that realizes $A$. 


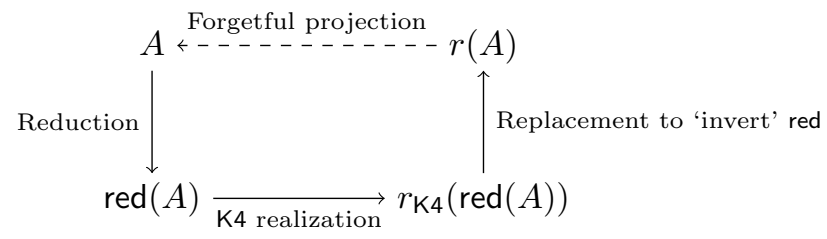

Theorem 34 (Realization for PAL). If $A$ is a theorem of PAL such that $A$ does not contain $\square$ operators within announcements, then for any properly annotated version $B$ of $A$, there is a realization function $r$ such that $r(B)$ is provable in JPAL.

Remark 35. It is not clear how to generalize our proof to all theorems of PAL. The problem is that the reduction of $[\square A](C \rightarrow D)$ produces two copies of $\square$ of opposite polarities. Those two occurrences of $\square$ may then be realized by different terms, and we lack methods of merging terms of opposite polarities in order to 'invert' the reduction.

Remark 36. Unfortunately, adapting this proof to OPAL presents certain challenges. The problem is that in order to 'invert' the reduction from PAL to K4, we need to apply replacement also in negative positions. This is only possible because in the update axiom (2) of JPAL, we have the same justification term on both sides of the equivalence. If, like in OPAL, we work with update operations $\Uparrow$ and $\Downarrow$ on terms, then we end up with different terms in the update axioms, which prevents the use of Fitting's replacement at negative positions.

\section{Conclusion}

This paper presents OPAL, a dynamic justification logic that includes term operators that reflect public announcements. One of OPAL's update axioms is

$$
[\sigma] t:(A \rightarrow[A] B) \rightarrow[\sigma][A] \Uparrow t: B,
$$

which we used in Example 4 to derive $\vdash[p] \Uparrow\left(c_{1} \cdot c_{2}\right): p$. The presence of $\Uparrow$ in the term $\Uparrow\left(c_{1} \cdot c_{2}\right)$ clearly points out that this evidence for $p$ is contingent on a prior public announcement.

For the semantics, we employ a combination of epistemic models from justification logic and simple model transformations from dynamic epistemic logics where the agent considers worlds that are inconsistent with the announcement as impossible. We show that OPAL is sound and complete with respect to this semantics.

We develop a realization method for dynamic justification logics and establish a partial realization theorem stating that JPAL realizes all the theorems of PAL that do not contain modalities within announcements. Finally, we discuss why our realization result does not easily transfer to OPAL. It should be noted that our realization method does not rely on cut elimination in PAL, the logic being realized. Its constructiveness, however, depends on cut elimination in $\mathrm{K} 4$, to which PAL is reducible. 
Acknowledgments We would like to thank Bryan Renne and Joshua Sack for introducing us to justified dynamic epistemic logic and for many valuable discussions. We thank the anonymous referees for their comments that helped improve the paper. We also thank Galina Savukova for editing this text.

\section{References}

1. S. N. Artemov. Justified common knowledge. Theoretical Computer Science, 357(13):4-22, July 2006.

2. S. N. Artemov. The logic of justification. The Review of Symbolic Logic, 1(4):477513, Dec. 2008.

3. S. N. Artemov. Tracking evidence. In A. Blass, N. Dershowitz, and W. Reisig, editors, Fields of Logic and Computation, Essays Dedicated to Yuri Gurevich on the Occasion of His 70th Birthday, volume 6300 of Lecture Notes in Computer Science, pages 61-74. Springer, 2010.

4. S. N. Artemov and R. Kuznets. Logical omniscience as a computational complexity problem. In A. Heifetz, editor, Theoretical Aspects of Rationality and Knowledge, Proceedings of the Twelfth Conference (TARK 2009), pages 14-23, Stanford University, California, July 6-8, 2009. ACM.

5. V. N. Brezhnev. On explicit counterparts of modal logics. Technical Report CFIS 2000-05, Cornell University, 2000.

6. K. Brünnler, R. Goetschi, and R. Kuznets. A syntactic realization theorem for justification logics. In L. Beklemishev, V. Goranko, and V. Shehtman, editors, Advances in Modal Logic, Volume 8, pages 39-58. College Publications, 2010.

7. S. Bucheli, R. Kuznets, B. Renne, J. Sack, and T. Studer. Justified belief change. In $\mathrm{X}$. Arrazola and M. Ponte, editors, LogKCA-10, Proceedings of the Second ILCLI International Workshop on Logic and Philosophy of Knowledge, Communication and Action, pages 135-155. University of the Basque Country Press, 2010.

8. S. Bucheli, R. Kuznets, and T. Studer. Justifications for common knowledge. To appear in the Journal of Applied Non-Classical Logics, 2011.

9. M. Fitting. The logic of proofs, semantically. Annals of Pure and Applied Logic, 132(1):1-25, Feb. 2005.

10. M. Fitting. The realization theorem for S5, a simple, constructive proof. In A. Gupta and J. van Benthem, editors, Proceedings of the Second Indian Conference on Logic and Its Relationship with Other Disciplines, 2009. Forthcoming.

11. M. Fitting. Realizations and LP. Annals of Pure and Applied Logic, 161(3):368387, Dec. 2009.

12. J. Gerbrandy. Bisimulations on Planet Kripke. PhD thesis, Institute for Logic, Language, and Computation, University of Amsterdam, 1999.

13. J. Gerbrandy and W. Groeneveld. Reasoning about information change. Journal of Logic, Language and Information, 6(2):147-169, Apr. 1997.

14. J. Plaza. Logics of public communications. Synthese, 158(2):165-179, Sept. 2007. Reprinted from M. L. Emrich et al., editors, Proceedings of the 4th International Symposium on Methodologies for Intelligent Systems (ISMIS '89), pages 201-216. Oak Ridge National Laboratory, ORNL/DSRD-24, 1989.

15. B. Renne. Dynamic Epistemic Logic with Justification. PhD thesis, CUNY Graduate Center, May 2008. 
16. B. Renne. Evidence elimination in multi-agent justification logic. In A. Heifetz, editor, Theoretical Aspects of Rationality and Knowledge, Proceedings of the Twelfth Conference (TARK 2009), pages 227-236, Stanford University, California, July 6-8, 2009. ACM.

17. B. Renne. Public communication in justification logic. Journal of Logic and Computation, Advance Access, July 2010.

18. H. van Ditmarsch, W. van der Hoek, and B. Kooi. Dynamic Epistemic Logic, volume 337 of Synthese Library. Springer, 2007.

\section{Appendix}

Proof of Lemma 20. As usual, the proof is by induction on the length of the derivation of $A$ in OPAL. We only show the most interesting cases for the axioms that relate announcements and justifications.

1. Independence. $\mathcal{M}, w \Vdash[\sigma] p$ iff $\mathcal{M}_{\sigma}, w \Vdash p$ iff $w \in \nu_{\sigma}(p)$ iff $w \in \nu(p)$ iff $\mathcal{M}, w \Vdash p$.

2. Update $\Uparrow . \mathcal{M}, w \Vdash[\sigma] t:(A \rightarrow[A] B)$ is equivalent to the conjunction of

$$
\begin{gathered}
A \rightarrow[A] B \in \mathcal{E}^{\sigma}(w, t) \quad \text { and } \\
\mathcal{M}_{\sigma}, v \Vdash A \rightarrow[A] B \text { for all } v \text { with } R_{\sigma}(w, v) .
\end{gathered}
$$

By the condition (3) on $\mathcal{E}$ from Def. 11, we obtain that (6) implies

$$
B \in \mathcal{E}^{\sigma, A}(w, \Uparrow t)
$$

Moreover, (7) is equivalent to

$$
\mathcal{M}_{\sigma}, v \Vdash A \text { implies } \mathcal{M}_{\sigma, A}, v \Vdash B \text { for all } v \text { with } R_{\sigma}(w, v) \text {, }
$$

which, in turn, is equivalent to

$$
\mathcal{M}_{\sigma, A}, v \Vdash B \text { for all } v \text { with } R_{\sigma, A}(w, v) \text {. }
$$

The conjunction of this and (8) is equivalent to $\mathcal{M}_{\sigma, A}, w \Vdash \Uparrow t: B$, or, equivalently, $\mathcal{M}, w \Vdash[\sigma][A] \Uparrow t: B$.

3. Update $\Downarrow$. $\mathcal{M}, w \Vdash[\sigma][A] t: B$ is equivalent to the conjunction of (10) and $B \in \mathcal{E}^{\sigma, A}(w, t)$ By the condition (4) on $\mathcal{E}$ from Def. 11, the latter implies

$$
A \rightarrow[A] B \in \mathcal{E}^{\sigma}(w, \Downarrow t)
$$

Moreover, as noted in the previous case, (10) is equivalent to (7). The conjunction of (7) and (11) is equivalent to $\mathcal{M}_{\sigma}, w \Vdash \Downarrow t:(A \rightarrow[A] B)$, or, equivalently, $\mathcal{M}, w \Vdash[\sigma] \Downarrow t:(A \rightarrow[A] B)$.

4. Iteration. First we show that

$$
R_{\sigma, A, B}=R_{\sigma, A \wedge[A] B}
$$


$R_{\sigma, A, B}(u, v)$ is equivalent to $R_{\sigma}(u, v)$ and $\mathcal{M}_{\sigma}, v \Vdash A$ and $\mathcal{M}_{\sigma, A}, v \Vdash B$. This is equivalent to $R_{\sigma}(u, v)$ and $\mathcal{M}_{\sigma}, v \Vdash A \wedge[A] B$, which, in turn, is equivalent to $R_{\sigma, A \wedge[A] B}(u, v)$, and thus (12) is established.

The case for iteration is now as follows: $\mathcal{M}, w \Vdash[\sigma][A][B] C$ if and only if $\mathcal{M}_{\sigma, A, B}, w \Vdash C$. By condition (5) on $\mathcal{E}$ from Def. 11 and by (12), this is equivalent to $\mathcal{M}_{\sigma, A \wedge[A] B}, w \Vdash C$, which, in turn, is equivalent to the statement $\mathcal{M}, w \Vdash[\sigma][A \wedge[A] B] C$.

To establish completeness, we need to know that the canonical model is a model.

Lemma 37 (Correctness of the Canonical Model). The canonical model is a model.

Proof. First, we observe that the set $W$ is non-empty: the set of all formulas that are true at world $w$ of the model from Example 13 is maximally consistent. We next show that $\mathcal{E}^{\sigma}$ is an evidence function on $(W, R)$ for each $\sigma$.

- Monotonicity. Assume $A \in \mathcal{E}^{\sigma}(w, t)$ and $R(w, v)$. We have $[\sigma] t: A \in w$. By introspection and normality, we get $[\sigma] ! t: t: A \in w$. By $R(w, v)$, we find $[\sigma] t: A \in v$. Thus, $A \in \mathcal{E}^{\sigma}(v, t)$.

- Axioms. For any $c: A$ derivable by $\mathrm{AN},[\sigma] c: A$ is also derivable for any $\sigma$. Hence, $[\sigma] c: A \in w$ and $A \in \mathcal{E}^{\sigma}(w, c)$.

- Application. Assume $A \rightarrow B \in \mathcal{E}^{\sigma}(w, t)$ and $A \in \mathcal{E}^{\sigma}(w, s)$. We then have $[\sigma] t:(A \rightarrow B) \in w$ and $[\sigma] s: A \in w$. By application and normality, we get $[\sigma] t \cdot s: B \in w$. Thus, $B \in \mathcal{E}^{\sigma}(w, t \cdot s)$.

- Sum and Introspection are shown as in the previous case, using the axioms of sum and introspection respectively.

Next we show condition (3) on $\mathcal{E}$ from Def. 11. We have $A \rightarrow[A] B \in \mathcal{E}^{\sigma}(w, t)$ if and only if $[\sigma] t:(A \rightarrow[A] B) \in w$. By the update $\Uparrow$ axiom, the latter implies $[\sigma][A] \Uparrow t: B \in w$, which is equivalent to $B \in \mathcal{E}^{\sigma, A}(w, \Uparrow t)$.

Conditions (4) and (5) are shown similarly using the update $\Downarrow$ axiom and the iteration axiom respectively.

Finally, we show that $R$ is transitive. Let $R(w, v)$ and $R(v, u)$. Whenever $[\sigma] t: A \in w$, by introspection and normality, $[\sigma] ! t: t: A \in w$. Then $[\sigma] t: A \in v$ since $R(w, v)$. And finally, $[\sigma] A \in u$ since $R(v, u)$. Thus, $R(w, u)$.

We will need a rank function on formulas to prove the Truth Lemma.

Definition 38 (Rank). The $\operatorname{rank} \operatorname{rk}(A)$ of a formula $A$ is defined as follows:

$$
\begin{aligned}
\operatorname{rk}(p):= & 1 \text { for each } p \in \operatorname{Prop}, \\
\operatorname{rk}(\neg A):=\operatorname{rk}(A)+1, & \operatorname{rk}(A \rightarrow B):=\max (\operatorname{rk}(A), \operatorname{rk}(B))+1, \\
\operatorname{rk}(t: A):=\operatorname{rk}(A)+1, & \operatorname{rk}([A] B):=(2+\operatorname{rk}(A)) \cdot \operatorname{rk}(B) .
\end{aligned}
$$

We immediately get the following lemma: 
Lemma 39 (Reductions Reduce Rank). For all formulas $A, B, C$ and all terms $t$, we have the following:

1. $\operatorname{rk}(A)>\operatorname{rk}(B)$ if $B$ is a proper subformula of $A$

2. $\operatorname{rk}([A] \neg B)>\operatorname{rk}(\neg[A] B) \quad$ 3. $\operatorname{rk}([A](B \rightarrow C))>\operatorname{rk}([A] B \rightarrow[A] C)$

4. $\operatorname{rk}([A] t: B)>\operatorname{rk}(\Downarrow t:(A \rightarrow[A] B)) \quad$ 5. $\operatorname{rk}([A][B] C)>\operatorname{rk}([A \wedge[A] B] C)$

Proof of Lemma 23. Proof by induction on $\mathrm{rk}(D)$ and a case distinction on the structure of $D$. Let us only show the cases where $D$ is of the form $[A] B$. The other cases are standard and follow easily from the maximal consistency of $w$ and the definition of the canonical model.

1. $D=[A] p$. Suppose $[A] p \in w$. By the independence axiom, this is equivalent to $p \in w$ for a formula of a lower rank than $[A] p$. By the induction hypothesis, this is equivalent to $\mathcal{M}, w \Vdash p$, which is equivalent to $\mathcal{M}, w \Vdash[A] p$ by the soundness of the independence axiom.

2. $D=[A] \neg B$. Suppose $[A] \neg B \in w$. By the functionality axiom, this is equivalent to $\neg[A] B \in w$ for a formula of a lower rank than $[A] \neg B$. By the induction hypothesis, this is equivalent to $\mathcal{M}, w \Vdash \neg[A] B$, which is equivalent to $\mathcal{M}, w \Vdash[A] \neg B$ by the soundness of the functionality axiom.

3. $D=[A](B \rightarrow C)$ is shown similarly using the normality axiom.

4. $D=[A] t: B$. Suppose $[A] t: B \in w$. By the update $\Downarrow$ axiom, we then have $\Downarrow t:(A \rightarrow[A] B) \in w$ for a formula of a lower rank than $[A] t: B$. By the induction hypothesis, this is equivalent to $\mathcal{M}, w \Vdash \Downarrow t:(A \rightarrow[A] B)$, which implies, in particular, that $\mathcal{M}, v \Vdash A \rightarrow[A] B$ whenever $R(w, v)$. Equivalently, $\mathcal{M}, v \Vdash[A] B$ whenever $R(w, v)$ and $\mathcal{M}, v \Vdash A$. Equivalently, $\mathcal{M}_{A}, v \Vdash B$ whenever $R_{A}(w, v)$. In addition, by the definition of $\mathcal{E}$, we have $B \in \mathcal{E}^{A}(w, t)$. To summarize, we have $\mathcal{M}_{A}, w \Vdash t: B$. In other words, $\mathcal{M}, w \Vdash[A] t: B$.

Suppose $[A] t: B \notin w$. By the definition of $\mathcal{E}$, we have $B \notin \mathcal{E}^{A}(w, t)$. Hence, $\mathcal{M}_{A}, w \nVdash t: B$. In other words, $\mathcal{M}, w \nVdash[A] t: B$.

5. $D=[A][B] C$. Suppose $[A][B] C \in w$. By the iteration axiom, this is equivalent to $[A \wedge[A] B] C \in w$ for a formula of a lower rank than $[A][B] C$. By the induction hypothesis, this is equivalent to $\mathcal{M}, w \Vdash[A \wedge[A] B] C$, which, by the soundness of the iteration axiom, is equivalent to $\mathcal{M}, w \Vdash[A][B] C$.

Proof of Theorem 24. Soundness was already shown in Lemma 20. For completeness, consider the canonical model $\mathcal{M}=(W, R, \mathcal{E}, \nu)$ and assume that $\nvdash A$. Then $\{\neg A\}$ is consistent and, hence, contained in some maximal consistent set $w \in W$. By Lemma 23, it follows that $\mathcal{M}, w \Vdash \neg A$ and, hence, that $\mathcal{M}, w \nVdash A$. Since $\mathcal{M}$ is a model (Lemma 37 ), we have shown that $\nvdash A$ implies $\nVdash A$. Completeness follows by contraposition.

Proof of Corollary 25. Assume $\vdash A$. By soundness, $\Vdash A$. Therefore, $\mathcal{M} \Vdash A$ for all models $\mathcal{M}$. In particular, $\mathcal{M}_{B}, w \Vdash A$ for all models of the form $\mathcal{M}_{B}$ and worlds $w$ in them. Thus, we obtain $\mathcal{M}, w \Vdash[B] A$ for all $\mathcal{M}, w$. By completeness, we conclude $\vdash[B] A$. 
Proof of Theorem 27. The proof is by induction on the length of the derivation of $A$ in OPAL. For the base case, simply observe that the forgetful projection of each axiom of OPAL is derivable in PAL. The rest is straightforward.

Proof sketch of Theorem 33. This theorem follows from the JPAL version of [11, Theorem 4.3]. For space considerations we only show the additional case in the algorithm that constructs $\left\langle r_{\varphi}, \sigma_{\varphi}\right\rangle$ and omit the correctness proof.

Announcement case: $\varphi(P)$ is $[\theta] \eta(P)$, where $\theta$ is $\square_{n}$-free and $\left\langle r_{\eta}, \sigma_{\eta}\right\rangle$ has been constructed. Since subformulas of $\theta$ are neither positive nor negative, $P$ does not occur in $\theta$. Set $\sigma_{\varphi}=\sigma_{\eta}$ and define $r_{\varphi}$ as follows:

$$
r_{\varphi}(n)= \begin{cases}r_{\eta}(n) & \text { if } \square_{n} \text { occurs in } \eta(B) \\ r_{1}(n) & \text { otherwise. }\end{cases}
$$

Proof of Theorem 34. Let $A$ be a formula of Fml $\square,[\cdot]$ that does not contain $\square$ operators within announcements such that PAL $\vdash A$. By Lemma 18, we find $\mathrm{PAL} \vdash \operatorname{red}(A)$ with no modalities occurring within announcements throughout the reduction process (see Def. 17). By the soundness of PAL, $\operatorname{red}(A)$ is a valid formula of $\mathrm{Fml} \square$, and by completeness of $\mathrm{K} 4$, we obtain $\mathrm{K} 4 \vdash \operatorname{red}(A)$.

Let $B$ be a properly annotated version of $\operatorname{red}(A)$. By Theorem 32 , there exists a realization function $r_{\mathrm{K} 4}$ with $\mathrm{J} 4 \vdash r_{\mathrm{K} 4}(B)$ such that $r_{\mathrm{K} 4}$ is non-self-referential on variables over $B$. Since J4 is a subsystem of JPAL, we also have JPAL $\vdash r_{\mathrm{K}_{4}}(B)$.

Now we iteratively 'invert' the reduction steps performed by red. Let us show case 6 in Definition 17. Let $E(q)$ be a formula with one occurrence of $q$ such that $E([C] \square D)$ has been reduced to the formula $E(\square(C \rightarrow[C] D))$, of which we let $E^{\prime}\left(\square_{i}\left(C \rightarrow[C] D^{\prime}\right)\right)$ be a properly annotated version. Note that $C$ does not contain any modalities and, hence, does not require any annotations. By induction hypothesis, there is a realization function $r_{1}$ such that

$$
\text { JPAL } \vdash r_{1}\left(E^{\prime}\left(\square_{i}\left(C \rightarrow[C] D^{\prime}\right)\right)\right),
$$

which is non-self-referential on that formula. By the JPAL update axiom (2), we have JPAL $\vdash r_{1}\left(\square_{i}\left(C \rightarrow[C] D^{\prime}\right) \leftrightarrow r_{1}\left([C] \square_{i} D^{\prime}\right)\right.$. Whether $q$ occurs positively or negatively in $E^{\prime}(q)$, applying the replacement theorem yields a realization/substitution pair $\langle r, \sigma\rangle$ such that

$$
\mathrm{JPAL} \vdash r_{1}\left(E^{\prime}\left(\square_{i}\left(C \rightarrow[C] D^{\prime}\right)\right)\right) \sigma \rightarrow r\left(E^{\prime}\left([C] \square_{i} D^{\prime}\right)\right),
$$

with $r$ being non-self-referential on variables over $E^{\prime}\left([C] \square_{i} D^{\prime}\right)$. By the substitution lemma, (13) implies JPAL $\vdash r_{1}\left(E^{\prime}\left(\square_{i}\left(C \rightarrow[C] D^{\prime}\right)\right)\right) \sigma$, from which JPAL $\vdash r\left(E^{\prime}\left([C] \square_{i} D^{\prime}\right)\right)$ follows by modus ponens.

After having 'inverted' all reduction steps, we end up with a properly annotated version $F$ of $A$ and a realization function $r$ such that $\vdash r(F)$. 